\title{
Measuring Inhomogeneity in Spatial Distributions
}

\author{
Udo Schilcher\#, Michael Gyarmati\#, Christian Bettstetter\#, Yun Won Chung*, and Young Han Kim* \\ \# Mobile Systems Group, University of Klagenfurt, Austria, <firstname.lastname>@uni-klu.ac.at \\ * School of Electronic Engineering, Soongsil University, Korea, \{ywchung@,yhkim@dcn.\}ssu.ac.kr
}

\begin{abstract}
The spatial distribution of nodes in wireless networks has important impact on network performance properties, such as capacity and connectivity. Although random sample models based on a uniform distribution are widely used in the research community, they are inappropriate for scenarios with clustered, inhomogeneous node distribution.

This paper proposes a well-defined measure for the level of inhomogeneity of a node distribution. It is based on the local deviation of the actual value of the density of nodes from its expected value. Desired properties of the measure are defined and mathematically proven to be fulfilled. The inhomogeneity measure is also compared to human perception of inhomogeneity gained via an online survey. The results reveal that the measure well fits human perception, although there are notable deviations if linear operations are applied.
\end{abstract}

Index Terms-Wireless networks, simulation, modeling, inhomogeneous spatial distribution, inhomogeneity measure.

\section{INTRODUCTION AND MOTIVATION}

Simulation-based analysis of wireless networks requires a set of modeling assumptions describing the behavior of the system and its environment. For system-level analysis, some of the important issues include channel models, speech and data traffic models, and mobility models. Much research has been devoted to these building blocks, and many models exist for different scenarios and technologies (see, e.g. [1][5]). Another modeling class, however, has received much less attention in the wireless research community so far: the spatial distribution of the devices. It states how many nodes are located per unit area for each location of the system area, thus having significant impact on various network properties, such as connectivity, capacity, and medium access behavior.

A frequently used method to distribute devices is to take random samples from a two-dimensional uniform distribution. Such an approach is, however, unrealistic in many scenarios. It is especially inappropriate for sparsely-connected, delaytolerant networks [6] and wireless community networks [7], as these networks typically consist of clustered node distributions. The current research of the authors is thus devoted to the development of well-described and well-analyzed models for non-uniform (inhomogeneous) spatial node distributions. The overall goal is to enable fellow researchers to easily use inhomogeneous distributions with known stochastic properties in their simulations.

A possible way to use inhomogeneous node distributions is to collect location data in the real world (see, e.g. [8], [9]) and apply these data in simulations. Such actual location of nodes is, however, difficult to obtain accurately without additional infrastructure or GPS receivers. Therefore, many data gathering projects do not collect the location data itself but derive related data, such as number of neighbors in range. Even if real node distributions are available, the obtained simulation results are restricted to the investigated environment (e.g. a university campus) and are not of generic applicability.

An alternative way to use inhomogeneous node distributions is to apply a synthetic, random model designed to generate distributions that show similar properties to the real environment. The advantage of such an approach - compared to real-world samples - is that we can generate many different spatial distributions to simulate on. Steps in this direction were made in [10], [11], and in the authors' paper [12]. The latter presented an algorithm to create a random inhomogeneous node distribution based on a neighborhood-dependent thinning of a homogeneous Poisson process. Nodes are initially placed uniformly, but then all nodes having less than $k$ neighbors in a given range $r$ are deleted. The inhomogeneity of the distribution can be tailored by the two parameters $k$ and $r$. The resulting distribution has well-defined stochastic properties (e.g. with respect to the nearest neighbor distance). These properties are also analyzed in [12]. The selection of the parameters $k$ and $r$ is, however, left to the user and depends on his or her subjective perception of inhomogeneity.

In this paper, we go one step further: we define an objective measure for the degree of inhomogeneity of a node distribution. The basic questions are: How can we quantify the inhomogeneity of a node distribution? Which properties should such a measure have? Our contribution is three-fold: First, we are not aware of any well-defined measure for inhomogeneity of node distributions in mobile communications. As the presented measure is independent of previous work, it gives a generic measure to characterize a node distribution. Second, the measure is useful for creating node distributions as described in [12]. A researcher is no longer forced to set the parameters $k$ and $r$, but just defines the degree of inhomogeneity, which will then be translated into appropriate values of $k$ and $r$. Third, such a measure is of practical use if real-world location data has been collected. We can measure the inhomogeneity of the real-world distribution and then synthetically generate random node distributions with a similar level of inhomogeneity.

This paper is organized as follows: In Section II we define required properties for an inhomogeneity measure. Section III describes our approach and proves that it satisfies the given properties. A comparison with the human perception of inhomogeneity is presented in Section IV. Section V gives an overview of related work. Finally, Section VI concludes and gives an outlook on further research in this field. 


\section{InHOMOGENEITY MEASURE REQUIREMENTS}

Before defining an inhomogeneity measure, let us first discuss the following basic questions: What are general requirements on such a measure? What are important aspects when defining a measure for spatial distributions? We list a number of properties that an inhomogeneity measure should fulfill. Some of these properties are necessary to get a reasonable measure, others are introduced for a simple use of the measure.

An inhomogeneity measure should fulfill the following three properties: First, the measure is in the range between " 0 " and " 1 ", with " 0 " indicating a perfectly uniform, and "1" indicating an extremely non-uniform distribution. This property is mainly for an easy usage of the measure. Second, the measure is independent of the number of nodes ${ }^{1}$ and the size of the area in which the nodes are distributed. Third, the measure is independent of linear operations (e.g. moving, scaling, mirroring, rotating), as such transformations do not have any influence on the degree of inhomogeneity of a distribution. Furthermore, the measure supports wrap-around distance metrics to avoid border effects (see, e.g. [12]).

\section{A Grid-BASED Inhomogeneity Measure}

\section{A. Definitions}

Given is a rectangular area $A$ with side lengths $a$ and $b$. An $s$-segmentation of the area $A$ is a subdivision of both edges of $A$ into $s$ parts of same length. In other words, the area is subdivided into a grid with $s^{2}$ rectangular subareas of same size. These subareas are called $A_{i}$ with $i=1,2, \ldots, s^{2}$. The number of nodes located in a subarea $A_{i}$ is called $m_{i}$.

An offset $(x, y) \in\left[0, \frac{a}{s}\right) \times\left[0, \frac{b}{s}\right)$ of an $s$-segmentation is defined as follows. In the horizontal direction, the entire grid is moved to the right by $x$; the parts of the segmentation that leave $A$ at the right border are assumed to be inserted at the left border of $A$. The value $y$ plays the same role for the vertical direction. The upper bounds for $x$ and $y$ are due to symmetry. For a particular offset $(x, y)$, the number of nodes in a subarea $A_{i}$ is called $m_{i,(x, y)}$. An $s^{\prime}$-segmentation is called a refinement of an $s$-segmentation if and only if $s^{\prime}>s$.

\section{B. Derivation of the Inhomogeneity Measure}

If we distribute $n$ nodes uniformly randomly on the area $A$, the expected number of nodes in each subarea $A_{i}$ is

$$
\bar{m}(s):=\bar{m}_{i}(s)=\frac{n}{s^{2}} \forall i .
$$

The deviation of the actual number of nodes $m_{i}$ in an area $A_{i}$ from this expected value $\bar{m}$ is an indicator for the local inhomogeneity of the spatial distribution. Hence, we define the inhomogeneity of an $s$-segmentation with offset $(x, y)$ as

$$
h_{(x, y)}(s):=\frac{1}{2 n} \sum_{i=1}^{s^{2}}\left|m_{i,(x, y)}-\bar{m}(s)\right|,
$$

with the normalization factor $\frac{1}{2 n}$ (see Property 2 below).

\footnotetext{
${ }^{1}$ More nodes should not result in higher inhomogeneity values. As no reference measure for inhomogeneity exists it is impossible to create different distributions with various numbers of nodes but the same inhomogeneity to verify this property.
}

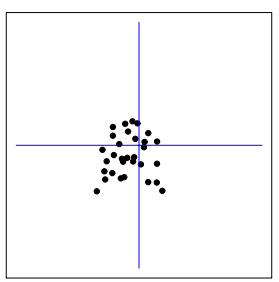

(a) Centered Cluster

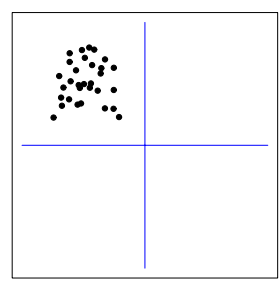

(b) Moved Cluster
Fig. 1. Different Inhomogeneity Values for a Moved Cluster.

As illustrated in Figure 1, the value of $h_{(x, y)}(s)$ depends on the offset. Fig. 1(a) depicts a node cluster that is centered; Fig. 1(b) shows the same cluster moved to the upper left corner. Both figures contain a 2-segmentation of the area. If the cluster is centered, all four subareas contain approximately the same number of nodes. This would give you the impression that there is a homogeneous distribution. This effect must be avoided, as a good inhomogeneity measure is independent of linear operations of the entire set of nodes. This can be done by choosing an offset that maximizes the inhomogeneity value for a given $s$-segmentation (as in Fig. 1(b)). Thus, we define the inhomogeneity of an $s$-segmentation as

$$
h(s):=\max _{(x, y)} h_{(x, y)}(s) .
$$

The inhomogeneity $h(s)$ can be interpreted as a measure with an adjustable locality. If the $s$-segmentation is being refined, more local deviations to an idealized homogeneous distribution are taken into account. At some point when the segmentation is sufficiently refined, even for a homogeneous distribution, the value is increasing due to local variations. For inhomogeneous distributions these deviations can be "seen" by this measure with a much less refined segmentation, e.g. if all nodes are in the upper left quarter of $A$, even a 2-segmentation indicates complete inhomogeneity. Thus, high values of $h(s)$ for rough $s$-segmentations indicate an inhomogeneous distribution, whereas small values indicate a homogeneous distribution. For more refined $s$-segmentations the difference between these two situations is decreasing. At a certain point (e.g. if there is no subarea containing more than one node) there is no difference between a homogeneous and an inhomogeneous distribution.

We reach our final goal: the definition of an inhomogeneity measure that is independent of the area segmentation. For this purpose, we generate area segmentations with $s=2,4,8, \ldots, 2^{r}(r \in \mathbb{N})$ until we have reached a certain segmentation in which each subarea contains at most one node. For each segmentation, we compute $h(s)$ and build a weighted sum in which a more refined segmentation ( $s$ high) gets a lower weight. The inhomogeneity $h$ of a node distribution is thus defined as

$$
\begin{aligned}
h & :=\sum_{j=1}^{r} w^{1-j} h\left(2^{j}\right) \\
& =\frac{1}{2 n} \sum_{j=1}^{r} w^{1-j} \max _{(x, y)} \sum_{i=1}^{2^{2 j}}\left|m_{i,(x, y)}-\frac{n}{2^{2 j}}\right| .
\end{aligned}
$$


The constant $w$ is selected in a way to achieve $h \leq 1$ (see Section III-D2: $w \approx 4.79129$ ). The upper bound $r$ in the sum is chosen in a way that each subarea in a $2^{r}$-segmentation contains at most one node. If two or more nodes have exactly the same position, the sum goes to infinity. This infinite sum converges for all $w>1$.

\section{Basic Properties of $h(s)$}

In the following we prove some basic properties of the inhomogeneity measure $h(s)$.

Property 1: The inhomogeneity $h_{(x, y)}(s)$ never decreases for a refinement of the segmentation, i.e.,

$$
h_{(x, y)}\left(s^{\prime}\right) \geq h_{(x, y)}(s) \quad \forall s \text { dividing } s^{\prime} .
$$

Proof: If $s$ divides $s^{\prime}$ there exists a set of subareas $A_{j}^{\prime}$ with $j=1, \ldots, t$ of the $s^{\prime}$-segmentation for each $A_{i}$ being a subarea of the $s$-segmentation such that $\bigcup_{j=1}^{t} A_{j}^{\prime}=A_{i}$. Let $m_{j,(x, y)}^{\prime}$ be the number of nodes located in $A_{j}^{\prime}$. For a given $A_{i}$ we get $\left|m_{i,(x, y)}-\bar{m}(s)\right|=\left|\sum_{j=1}^{t} m_{j,(x, y)}^{\prime}-t \cdot \bar{m}\left(s^{\prime}\right)\right| \leq$ $\sum_{j=1}^{t}\left|m_{j,(x, y)}^{\prime}-\bar{m}\left(s^{\prime}\right)\right|$ due to the triangle inequality.

Property 2: Consider a distribution of $n$ nodes. If we refine a segmentation to infinitely small subareas, the inhomogeneity $h(s)$ converges to 1 , i.e., $\lim _{s \rightarrow \infty} h(s)=1$.

Proof: There exists an $s^{\prime}$ such that in no subarea of the corresponding $s^{\prime}$-segmentation two nodes with different positions are located. In other words, if there are two or more nodes in the same subarea, they have exactly the same position. Let $m_{i}$ with $i=0, \ldots, n$ denote the number of subareas containing $i$ nodes. Then we have

$$
h\left(s^{\prime}\right)=\frac{1}{2 n}\left(\sum_{i=1}^{n} m_{i}\left|\frac{n}{s^{2}}-i\right|+m_{0}\left|\frac{n}{s^{2}}-0\right|\right) .
$$

We use the fact that $m_{0}=s^{2}-\sum_{i=1}^{n} m_{i}$, let $s^{\prime} \rightarrow \infty$, and get

$$
\lim _{s^{\prime} \rightarrow \infty} h\left(s^{\prime}\right)=\frac{1}{2 n}(\underbrace{\sum_{i=0}^{n}\left|-m_{i} \cdot i\right|}_{=n}+n)=1 .
$$

This property shows that the inhomogeneity $h(s)$ converges to a fixed value for a sufficient segmentation of the area. This convergence holds independently of the distribution. The speed of convergence depends, however, on the distribution. The inhomogeneity $h$, which is independent of the segmentation, is higher for inhomogeneous distributions. This is due to the larger weights of the first summands.

\section{Minimum and Maximum of $h$}

In this section, we investigate which distributions minimize and maximize the inhomogeneity measure $h$. This insight is important to interpret a given inhomogeneity value. Obviously $h(s) \geq 0$ holds for all distributions and all $s$-segmentations.
1) Minimum Value: The inhomogeneity measure should be small for node distributions in which the nodes are "equally scattered" over the entire area. Let us consider the extreme case, namely a grid distribution: A spatial distribution of $n$ nodes is called a grid distribution if each subarea of the $\sqrt{n}$ segmentation contains exactly one node. (We require $\sqrt{n} \in$ $\mathbb{N}$.). In the following we show that a grid distribution yields a minimum inhomogeneity.

Property 3: A grid distribution of $2^{i}$ nodes, $i$ even, yields an inhomogeneity $h=0$.

Proof: We examine a $2^{j}$-segmentation of the area $A$ for $j=1,2, \ldots, \frac{i}{2}$. Since each subarea of the $2^{\frac{i}{2}}$-segmentation contains exactly one node and $\bar{m}\left(2^{\frac{i}{2}}\right)=1, h\left(2^{\frac{i}{2}}\right)=0$. For the $2^{\frac{i}{2}-1}$-segmentation each subarea therefore contains 4 nodes and the expected number of nodes per subarea is 4 . Thus we have again $h\left(2^{\frac{i}{2}-1}\right)=0$. If we apply this idea to all $j=$ $\frac{i}{2}-2, \ldots, 1$ we get $h\left(2^{j}\right)=0$ for all $j=1,2, \ldots, \frac{i}{2}$.

2) Maximum Value and Normalization: The inhomogeneity value should be large for node distributions in which the nodes are densely located in one subarea, and the remaining subareas are empty. In the extreme case, all nodes are located at the same position.

Property 4: If all nodes are located at the same position, the inhomogeneity $h$ reaches its maximum.

Proof: The sum reaches its maximum if and only if all summands do so. Therefore, we find the maximum of $h\left(2^{j}\right)$ for $j=1, \cdots, r$. This value is maximal if all $n$ nodes are located in one common subarea. Since this assumption must hold for arbitrary fine segmentations, the distribution giving the maximum $h$ has all $n$ nodes at the same location.

Now we compute the maximum value of $h$. If all $n$ nodes are located in a single subarea, we obtain from (1) and (2) the inhomogeneity

$$
h(s)=\frac{1}{2 n}\left[\left|n-\frac{n}{s^{2}}\right|+\left(s^{2}-1\right)\left|0-\frac{n}{s^{2}}\right|\right]=1-\frac{1}{s^{2}} .
$$

Substituting (6) with $s=2^{j}$ into (4) yields

$$
h=\sum_{j=1}^{\infty} w^{1-j}\left(1-\frac{1}{2^{2 j}}\right) \text {. }
$$

Since the two series $\sum_{j=1}^{\infty} w^{1-j}$ and $\sum_{j=1}^{\infty} \frac{w^{1-j}}{2^{2 j}}$ converge, we may compute their limits separately and get

$$
\begin{aligned}
h & =\sum_{j=1}^{\infty} w^{1-j}-\sum_{j=1}^{\infty} \frac{w^{1-j}}{2^{2 j}} \\
& =\frac{w}{w-1}-\frac{w}{4 w-1} \\
& =\frac{3 w^{2}}{(w-1)(4 w-1)} .
\end{aligned}
$$

To achieve normalized values for $h$, we select the weight $w$ such that we get

$$
0 \leq h \leq 1
$$

Thus, we set (8) equal to 1 and obtain

$$
w=\frac{5+\sqrt{21}}{2} \approx 4.79129 .
$$

Note that there is a second solution for $w$ that cannot be used as the sum in (7) would diverge. 


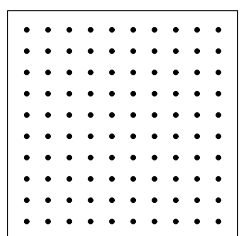

(a) $h=0.04$

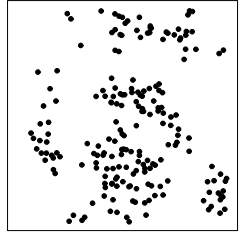

(g) $h=0.35$

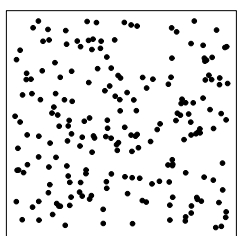

(b) $h=0.15$

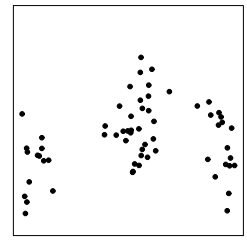

(h) $h=0.54$

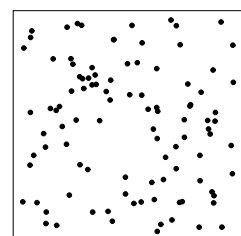

(c) $h=0.24$

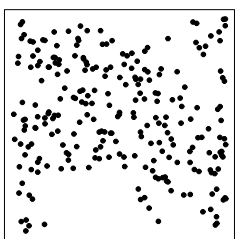

(d) $h=0.25$

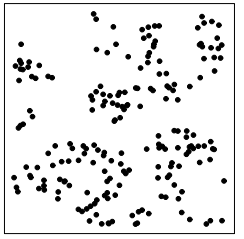

(j) $h=0.63$

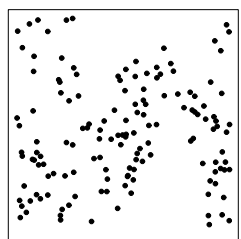

(e) $h=0.28$

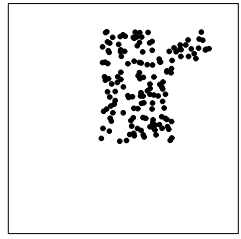

(k) $h=0.91$

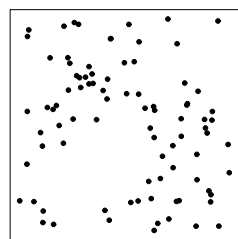

(f) $h=0.35$

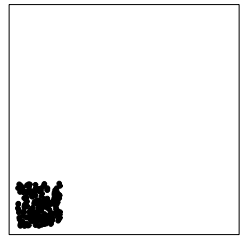

(1) $h=0.99$

Fig. 2. Inhomogeneity Values for Different Distributions.

\section{E. Visualization}

Figure 2 depicts different distributions and their corresponding inhomogeneity values $h$. Distribution 2(a) represents a grid distribution yielding a very low value of $h=0.04$. A random uniform distribution 2(b) also gets a low inhomogeneity value of $h=0.15$. By removing some nodes from a uniform distribution as in distributions 2(c)-2(g) some holes appear, and the inhomogeneity value increases. With even more compacted node clouds as in distributions $2(\mathrm{~h})-2(\mathrm{k})$ the value of the inhomogeneity becomes even higher. Finally, with a very dense distribution 2 (1) the inhomogeneity value $h=0.99$ almost reaches its theoretic maximum of $h=1$.

\section{COMPARISON With Human PERCEPTION OF INHOMOGENEITY}

We are now interested in the question as to whether the measure $h$ is in line with a human's intuition regarding the level of inhomogeneity in a distribution. In other words: If we compare two spatial distributions, do the measure $h$ and a human user make the same choice in selecting the more inhomogeneous distribution?

To investigate this issue, we conducted an online survey (mobile.uni-klu.ac.at/uniform/), asking researchers and students in Austria and South Korea to compare a set of 100 tuples of distributions with respect to their inhomogeneity. The system presents two pseudo-random distributions and asks the user to select the distribution which he or she finds more uniform, or to choose "similar" if she or he cannot decide. For both distributions the corresponding inhomogeneity measures are $h_{1}$ and $h_{2}$ are also calculated. The computer bases its "uniformity decision" on these values. At the end of each survey, the choices of the computer and the user are compared.

\section{A. General Results}

The results of a survey with 79 users are as follows. A fraction of $70 \%$ of the tuples are answered "correctly," i.e., the users classify the distributions in the same manner as the computer. In $18 \%$ of the tuples the users cannot decide although the distributions are different. In the remaining $12 \%$, the users' classification differs from that of the computer ("incorrect").

TABLE I

ANSWERS IN THE ONLINE SURVEY

\begin{tabular}{l|rrrrrrr} 
Class & 0.10 & 0.20 & 0.30 & 0.40 & 0.50 & 0.60 & 0.70 \\
\hline Correct & $42 \%$ & $69 \%$ & $90 \%$ & $94 \%$ & $95 \%$ & $94 \%$ & $93 \%$ \\
Undecided & $34 \%$ & $23 \%$ & $7 \%$ & $3 \%$ & $2 \%$ & $2 \%$ & $5 \%$ \\
Incorrect & $24 \%$ & $8 \%$ & $3 \%$ & $3 \%$ & $3 \%$ & $4 \%$ & $2 \%$
\end{tabular}

Table I lists a classification of all answers. The horizontal classes refer to the absolute difference $h_{\Delta}=\left|h_{1}-h_{2}\right|$. The numbers represent the percentage of correct, undecided, and incorrect answers in each class. Of all tuples with $h_{\Delta}<0.1$ approximately $42 \%$ are answered correctly, $34 \%$ are undecided; $24 \%$ are answered incorrectly. On one hand, this shows that for $h_{\Delta}<0.1$ the uniformity decision is difficult. On the other hand, for $h_{\Delta}>0.1$, the computer's objective decision well matches the human's subjective perception. In fact, $72 \%$ $(77 \%)$ of all incorrect (undecided) answers are given to tuples with $h_{\Delta}<0.1$. In conclusion, the inhomogeneity measure $h$ fits well the human perception of inhomogeneity.

\section{B. Perception of Linear Operations}

Another goal of the survey is to determine how human perception can cope with wrap-around distance and linear operations applied to distributions. For this purpose, two distributions that are identical but with linear operations applied are presented to the user several times between other random distributions.

An example is given in Figure 3. All figures depict the same distribution with $h=0.42$ but with different linear operations applied. Considering the first, left-most distribution as the original, the second one is moved by half the side length horizontally, the third one is transposed, and the fourth one is moved in such a way that the node with the most dense neighborhood is in the center. These distributions may look different to a human but the distribution is in fact the same. 


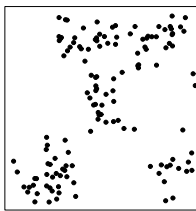

(a) Original

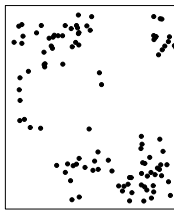

(b) Moved

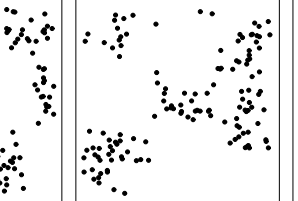

(c) Transposed

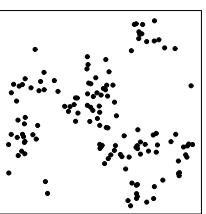

(d) Centered
Fig. 3. Effect of Linear Operations on Human Perception.

TABLE II

EFFECT OF LINEAR OPERATIONS IN ONLINE SURVEY.

\begin{tabular}{l|c} 
Linear Operation & Similar Answers (\%) \\
\hline Similar & $94 \%$ \\
Exchanged & $91 \%$ \\
Mirrored & $84 \%$ \\
Moved & $81 \%$ \\
Transposed & $83 \%$ \\
Centered & $80 \%$
\end{tabular}

Table II lists the percentage of similarly answered tuples after the linear operation was applied. Results show that presenting the same distributions again and exchanging the left and right image does have some impact on the users' decision which can be explained by inattentive users. Applying linear operations, however, results in more distinct differences.

\section{RELATED WORK}

Classical approaches in statistics use multiple hypothesis testing against a given distribution - e.g. the uniform distribution - to derive statements about the homogeneity of a distribution (see, e.g. [13]; [14]). Such hypothesis tests are, e.g. the chi-squared test and the Kolmogorov-Smirnov test. Since the output of a test can only be "yes" or "no," it does not provide any measure of the inhomogeneity in the sense of a real value between zero and one. The $p$-value of the test is also inadequate for such purposes.

Johansson describes how kurtosis can be used to measure the homogeneity of any property on a given area [15]. This approach is, however, not applicable to our scenario as it assumes some knowledge of the given distribution (e.g. the probability density function), which we do in general not know.

Spatial inhomogeneity measures are also needed and used in chemistry and physics. An overview of an aspect of work in that area is given in [16]. For example, Zwicky [17] uses an approach similar to (2). The measure does, however, not consider the offset and is thus variant to linear operations.

\section{CONClusions And Further Work}

In this paper we have presented an inhomogeneity measure that can be used to objectively rate the inhomogeneity of a spatial node distribution on a bounded area. Using this measure and our inhomogeneous spatial node distribution generation method from an earlier paper, an arbitrary number of similar inhomogeneous distributions for simulations can be generated. We have defined desired properties of an inhomogeneity measure and presented our approach along with proofs that it fulfills those properties. The analysis of an online survey showed that in most cases the objective measure corresponds to human perception. However, it also revealed issues with the human perception of inhomogeneity when linear operations are applied to the distributions.

Our inhomogeneity measure will also prove useful to assess mobility models. Basically, a mobility model should not change the basic type of node distribution, i.e. a sparse clustered network should not turn into a uniform distribution after a few steps. The random waypoint mobility model, for example, was shown to turn a uniform distribution into a distribution with high density in the center of the simulation area and zero density at the borders [4]. Using the inhomogeneity measure we are currently working on a mobility model that maintains the inhomogeneity of the initial distribution.

\section{ACKNOWLEDGMENT}

This work was supported by the ubiquitous Computing and Network (UCN) Project, the Ministry of Information and Communication (MIC) 21st Century Frontier R\&D Program in Korea. The Klagenfurt research group is supported by the Carinthian Economic Promotion Fund (KWF).

\section{REFERENCES}

[1] J. B. Andersen, T. S. Rappaport, and S. Yoshida, "Propagation measurements and models for wireless communications channels," IEEE Commun. Soc. Mag., vol. 33, no. 1, pp. 42-49, 1995.

[2] K. Leung, W. Massey, and W. Whitt, "Traffic models for wireless communication networks," IEEE J. Select. Areas Commun., vol. 12 no. 8, pp. 1353-1364, 1994.

[3] A. Klemm, C. Lindemann, and M. Lohmann, "Traffic modeling and characterization for UMTS networks," in Proc. IEEE Globecom, (San Antonio, TX, USA), 2001.

[4] C. Bettstetter, "Mobility modeling in wireless networks: Categorization, smooth movement, and border effects," ACM Mobile Computing and Commun. Rev., vol. 5, pp. 55-67, July 2001.

[5] T. Camp, J. Boleng, and V. Davies, "Mobility models for ad hoc network simulations," Wireless Commun. Mobile Comput., vol. 2, no. 5, pp. 483502, 2002.

[6] S. Farrell and V. Cahill, Delay- and Disruption-Tolerant Networking. Artech House, 2006.

[7] S. Jain and D. Agrawal, "Wireless community networks," IEEE Computer, vol. 36, pp. 90-92, Aug. 2003.

[8] N. Eagle and A. Pentland, "Reality mining: Sensing complex social systems," Personal and Ubiquitous Computing, vol. 10, pp. 255-268, May 2006.

[9] P. Hui, A. Chaintreau, J. Scott, R. Gass, J. Crowcroft, and C. Diot, "Pocket switched networks and human mobility in conference environments," in Proc. ACM SIGCOMM workshop on DTN, (New York, NY, USA), pp. 244-251, ACM Press, 2005.

[10] X. Liu and M. Haenggi, "Toward quasiregular sensor networks: Topology control algorithms for improved energy efficiency," IEEE Transactions on Parallel and Distributed Systems, vol. 17, pp. 975-986, 2006.

[11] D. Avidor and S. Mukherjee, "Hidden issues in the simulation of fixed wireless systems," ACM Wireless Networks, vol. 7, pp. 187-200, Apr. 2001.

[12] C. Bettstetter, M. Gyarmati, and U. Schilcher, "An inhomogeneous spatial node distribution and its stochastic properties," in Proc. ACM/IEEE Intern. Symp. on Modeling, Analysis, and Simulation of Wireless and Mobile Systems (MSWiM), (Chania, Crete Island, Greece), 2007.

[13] P. Vit, "Testing for homogeneity: the geometric distribution," Biometrika, vol. 61, no. 3, pp. 565-568, 1974.

[14] G. A. Baker, "Test of homogeneity for normal populations," The Annals of Mathematical Statistics, vol. 12, pp. 233-236, June 1941.

[15] J.-O. Johansson, "Measuring homogeneity of planar point-patterns by using kurtosis," Pattern Recognition Letters, vol. 21, pp. 1149-1156, 2000 .

[16] R. J. Piasecki, "A generalization of the inhomogeneity measure for point distributions to the case of finite size objects." http://arxiv.org/abs/condmat/0612401v2, 2006.

[17] F. Zwicky, Morphological Astronomy. Springer, 1957. 